\title{
The Long-Term Resetting of a Brainstem Pacemaker Nucleus by Synaptic Input: A Model for Sensorimotor Adaptation
}

\author{
Jörg Oestreich and Harold H. Zakon \\ Section of Neurobiology, University of Texas at Austin, Austin, Texas 78712
}

The cellular mechanisms behind sensorimotor adaptations, such as the adaptation to a sustained change in visual inputs by prism goggles in humans, are not known. Here we present a novel example of long-term sensorimotor adaptation in a well known neuroethological model, the jamming-avoidance response of a weakly electric fish. The adaptation is relatively long lasting, up to $9 \mathrm{hr}$ in vivo, and is likely to be mediated by NMDA receptors. We demonstrate in a brain slice preparation that the pacemaker nucleus is the locus of adaptation and that it responds to long-lasting synaptic stimulation with an increase in the postsynaptic spike frequency persisting for hours after stimulus termination. The mechanism for the neuronal memory behaves as an integrator, and memory duration and strength are quantitatively related to the estimated amount of synaptic stimulation. This finding is contrary to the idea that neurons respond solely to long-lasting synaptic input by turning down their intrinsic excitability. We show that this positive feedback at the cellular level actually contributes to a negative feedback loop at the organismic level if the entire neural circuit and the behavioral link are considered.

Key words: sensorimotor adaptation; postsynaptic plasticity; long-term; intrinsic excitability; activity increase; resetting; neural integrator; cellular memory; NMDA receptor; pacemaker; central pattern generator; brainstem; vertebrate; weakly electric fish; jamming avoidance response
An important aspect of sensorimotor control is sensorimotor adaptation: that is, generating a persistent adaptive change in motor output in response to a long lasting, stable change in sensory inflow. Sensorimotor adaptation is used by the nervous system to fine tune motor responses. A classic example occurs with the manipulation of visual inputs in humans or other animals by outfitting them with prism goggles (Held and Freedman, 1963). The offset in the visual input by a constant angle leads to an initial disorientation of the subjects, but, in minutes to tens of minutes, they learn to compensate for it. The adaptation becomes visible as a compensatory overshoot after taking off the prisms.

We present a novel example of sensorimotor adaptation in a simple vertebrate system, the electromotor circuit of the weakly electric fish, Apteronotus leptorhynchus. This circuit is particularly well understood, and, its behavioral output, the electric organ discharge (EOD), is easily quantified.

Weakly electric fish produce an electric field around their bodies with an electric organ. Distortions in the field lines caused by objects and other organisms are sensed and used for orientation. Individual fish maintain a highly regular EOD frequency. If two conspecifics with almost identical frequencies meet, signal interaction leads to jamming of their electrosensory systems. To avoid jamming, the higher-frequency individual shifts its frequency upward, away from the intruding frequency, the jammingavoidance response (JAR) (Watanabe and Takeda, 1963; Bullock et al., 1972a; Heiligenberg, 1986).

The EOD frequency is controlled by a pacemaker nucleus (PMn). The PMn receives input from two upstream nuclei, which

\footnotetext{
Received Jan. 24, 2002; revised June 12, 2002; accepted July 1, 2002.

This work was supported by National Institutes of Health Grant MH56535 (H.Z.). We thank G. T. Smith for helpful comments and Ying Lu for fish care.

Correspondence should be addressed to Jörg Oestreich, Section of Neurobiology, University of Texas at Austin, Patterson Laboratories, Austin, TX 78712. E-mail: joestreich@mail.utexas.edu.

Copyright (C) 2002 Society for Neuroscience $\quad 0270-6474 / 02 / 228287-10 \$ 15.00 / 0$
}

modulate the pacemaker firing rate and, therefore, EOD frequency (Heiligenberg et al., 1996). One input activates the JAR via NMDA receptors, and the other controls a behavior called "chirping" via AMPA receptors (Dye et al., 1989; Heiligenberg et al., 1996) (see Fig. 1). Because specific sensory stimuli result in the selective activation or coactivation of the JAR and chirping, the contribution of AMPA or NMDA receptors can be noninvasively assayed in behaving animals. Furthermore, in a PMn slice preparation with intact terminal portions of the afferent fibers, a fictive JAR can be induced by electrical stimulation.

The usual paradigm used to study the JAR is to give the fish a stimulus of up to $2 \mathrm{~min}$, after which its EOD frequency returns to its baseline value (Bullock et al., 1972a; Dye, 1987; Dulka and Maler, 1994; Heiligenberg et al., 1996; Takizawa et al., 1999). We found that prolonged exposure (30 min or $3 \mathrm{hr}$ ) of a fish to a jamming stimulus resulted in a sustained JAR. Stimulus termination revealed a long-term frequency elevation (LTFE) of the EOD frequency, which could last up to $9 \mathrm{hr}$ and during which the EOD frequency gradually relaxed back to baseline. This phenomenon is sensorimotor adaptation in that the firing frequency of the pacemaker was reset to minimize the effect of the jamming stimulus. LTFE was elicited by sensory stimuli that evoke a JAR and not those that induce bouts of chirping, supporting a role for NMDA, and not AMPA, receptors in the induction of LTFE. In vitro, activation of the afferent inputs to the PMn resulted in a fictive JAR followed by LTFE, suggesting that the PMn is the locus of sensorimotor adaptation in this system.

\section{MATERIALS AND METHODS}

Animals. Wild-caught individuals of A. leptorhynchus were obtained through different vendors and then housed in individual and community Plexiglas tanks in climate-controlled rooms with a circulating water system and a $12 \mathrm{hr}$ dark/light cycle. The temperature in the rooms was held stable between $\sim 26$ and $28^{\circ} \mathrm{C}\left( \pm 0.5^{\circ} \mathrm{C}\right)$, and the water conditions were relatively constant. Fish were fed with frozen brine shrimp every 

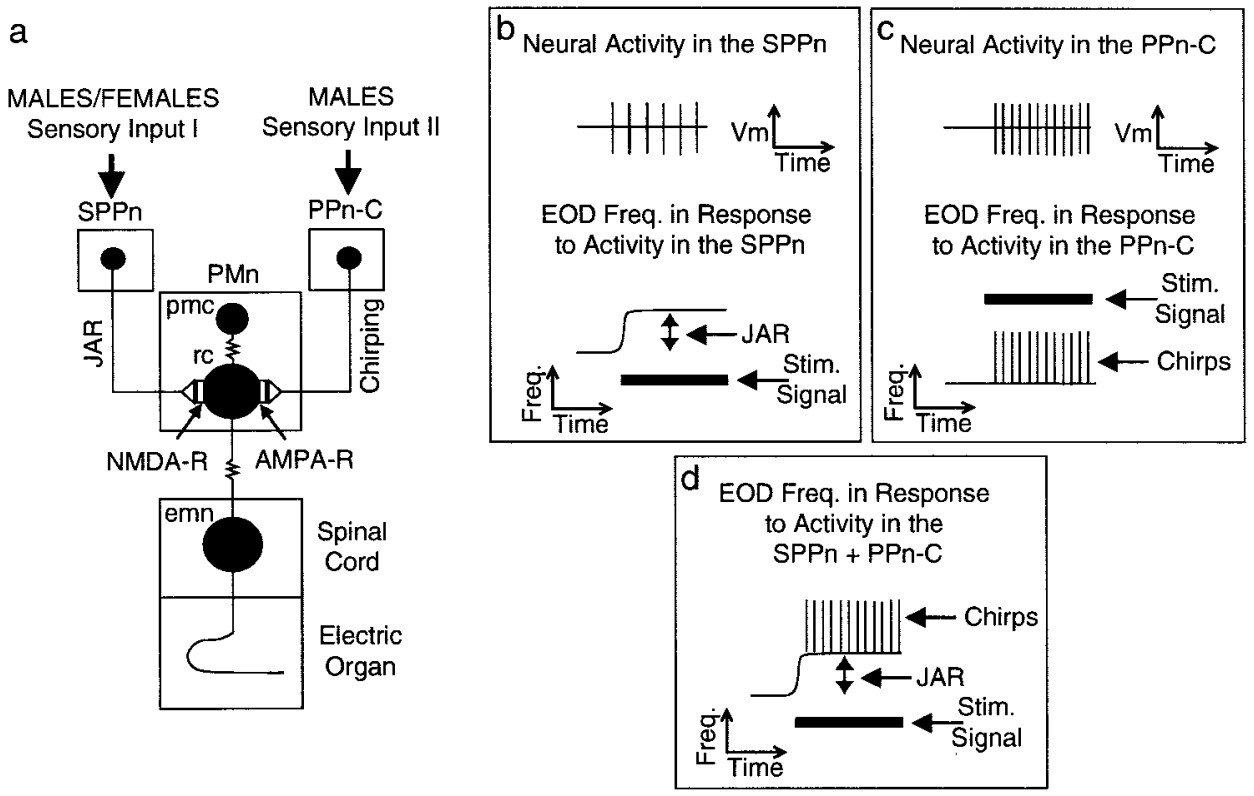

Figure 1. Schematic diagram of electromotor circuit with behavioral responses to neural activity in the prepacemaker nuclei. $a$, The base EOD frequency is set by a medullary PMn in a one-for-one manner. The PMn contains pacemaker ( $p m c)$ and relay cells $(r c)$, which are electrotonically coupled. A third neuronal cell type, possibly an interneuron with yet unknown function, is omitted for clarity (Smith et al., 2000). Relay cells provide electrotonic input to electromotor neurons $(\mathrm{emn})$ in the tail region of the spinal cord, which form the electric organ with their hypertrophied axons. The SPPn controls the JAR via NMDA receptor-carrying synapses with relay cells (Dye et al., 1989; Heiligenberg et al., 1996). $b$, This nucleus is normally quiescent and therefore does not exert any influence on the basic EOD frequency. In the presence of an appropriate jamming signal (e.g., frequency 3 $\mathrm{Hz}$ below EOD frequency), descending sensory input activates the nucleus and raises the PMn frequency, which in turn drives the electric organ to fire faster, the JAR. $c$, The PPn-C controls chirps, typically 10- to 15-msec-long EOD frequency rises. This nucleus uses AMPA receptors at its synapses with relay cells. This nucleus can be separately activated by nonjamming signals (e.g., $40 \mathrm{~Hz}$ above or below the EOD frequency). Chirping behavior is sexually dimorphic. Females rarely chirp under both stimulus conditions. $d$, In males, both stimulus situations evoke chirping. In a jamming situation, therefore, males display chirping behavior and JAR simultaneously (Zupanc and Maler, 1993; Dulka and Maler, 1994), whereas female fish show a pure JAR.

$2 \mathrm{~d}$. Individuals were allowed to acclimatize for 2 or more months before being used in experiments. Female fish used in the experiments ranged in size from 12.8 to $16.5 \mathrm{~cm}$, whereas male fish were 12.4 to $20.7 \mathrm{~cm}$ in length.

Behavioral testing. The behavioral experiments with individuals of $A$. leptorhynchus were performed by using a separate recording tank inside a Faraday cage. Fish were adapted to the conditions in the setup tank overnight, and experiments were only started if sufficient stability in the baseline EOD frequency was achieved. An artificial sine-wave signal produced by a computer (PII-based computer; Dell, Round Rock, TX), analog-to-digital (A/D) converted (DT3010/32; Data Translation, Marlboro, MA), and then attenuated (model 350D attenuator; HewlettPackard, Palo Alto, CA) was delivered through a pair of carbon rod electrodes across the body of the fish, whereas the fish's own EOD signal was recorded over platinum wire electrodes at both ends of the fish. The fish's body represents a dipole with the head region charged positive with respect to the tail region, which allowed the recording electrodes to be positioned at the body ends. The perpendicular arrangement of recording and stimulus electrodes avoided interference between EOD and stimulus signal. During the day, the nocturnal A. leptorhynchus can be found in shelters, e.g., plastic tubes in their home tanks. Therefore, a recording apparatus was used, which featured a plastic tube to keep the fish in a relatively stable position with respect to recording and stimulus electrodes (Dulka and Maler, 1994). To avoid temperature effects on the EOD frequency (Dunlap et al., 2000), the temperature in the setup tank was precisely controlled at an accuracy of at least $\pm 0.05^{\circ} \mathrm{C}$ using a separate temperature control system consisting of a programmable DC power supply (Kepco, Flushing, NY), heating pad (Frederick Haer Co., Bowdoinham, ME), and an industrial digital temperature controller (series 988; Watlow, St. Louis, MO), which measures the temperature in the tank near the recording tube by means of a resistance temperature detector probe. The temperature was separately monitored by an NISTtraced high accuracy thermistor thermometer (model 1504; Hart Scientific, American Fork, UT). The EOD signal was amplified (differential AC amplifier, model 1700; A \& M Systems, Sequim, WA), A/D converted (PCI-MIO-16E-4; National Instruments, Austin, TX), and digi- tally recorded (PII-based computer; Dell). The EOD frequency was calculated by using fast Fourier transform-based software [window size, 8192; sample rate, 2148 (written by J. Oestreich, based on Componentworks; National Instruments)] and constantly, together with the temperature reading from the thermometer, displayed on the computer monitor. The frequency then was offline corrected for temperature effects using a $q 10$ value of 1.61 (Dunlap et al., 2000). For the chirping experiments, the EOD was additionally captured continuously at a high sampling rate (125 $\mathrm{kHz}$ ) for later offline analysis with our own software. Every $50 \mathrm{msec}$, the frequency was averaged by using the number of threshold crossings of the EOD spikes. This resulted in a temporal resolution of $20 \mathrm{~Hz}$ and was sufficient to resolve chirps (Dye, 1987).

PMn slice preparation. Fish were anesthetized in $0.75 \%$ 2-phenoxyethanol (Sigma, St. Louis, MO) and then positioned on a bed of ice, and the brain was removed immediately. Continuously oxygenated $(95 \%$ $\mathrm{O}_{2}-5 \% \mathrm{CO}_{2}$ ) and ice-cold artificial CSF (ACSF) [in mM: $124 \mathrm{NaCl}, 2$ $\mathrm{KCl}, 1.25 \mathrm{KH}_{2} \mathrm{PO}_{4}, 1.1 \mathrm{MgSO}_{4}, 1.1 \mathrm{CaCl}_{2}, 16 \mathrm{NaHCO}_{3}$, and 10 glucose (Meyer, 1984)] was run through the scull cavity during the procedure to improve tissue healthiness. The brain was transferred to a Sylgard-coated Petri dish, which was filled with fresh oxygenated ice-cold ACSF, and then pinned down to the bottom, ventral side up. Under visual guidance through a dissection microscope, first the meninges were removed, and then a part of the ventral surface of the brainstem was carefully dissected from the rest of the brain using a pair of iridectomy scissors. The removed tissue spanned from a point in close proximity of the caudal aspect of the pacemaker nucleus to the pituitary fossa, which is located rostrally of the PMn. The thickness of the tissue slice was usually approximately one-half of the diameter of the brainstem. The pacemaker is visible as an ovoid, orange- to yellow-colored protrusion of the ventral surface of the medulla. The prepacemaker nuclei are located rostrally to the PMn, and the afferent fibers from the prepacemaker nuclei run close to the ventral surface of the medulla, shortly before they enter the PMn (Dye, 1988; Zupanc and Maler, 1997).

A Plexiglas tissue slice chamber [designed by R. Turner (University of Calgary, Calgary, Alberta, Canada) and L. Maler (University of Ottawa, Ottawa, Ontario, Canada)] was used to maintain the brain tissue 
a

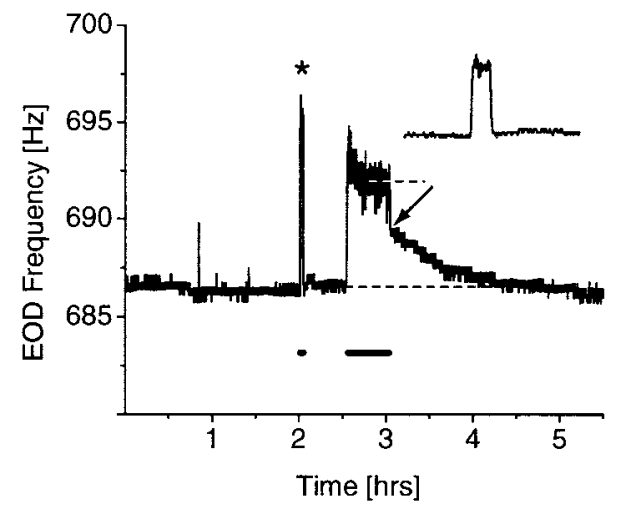

b

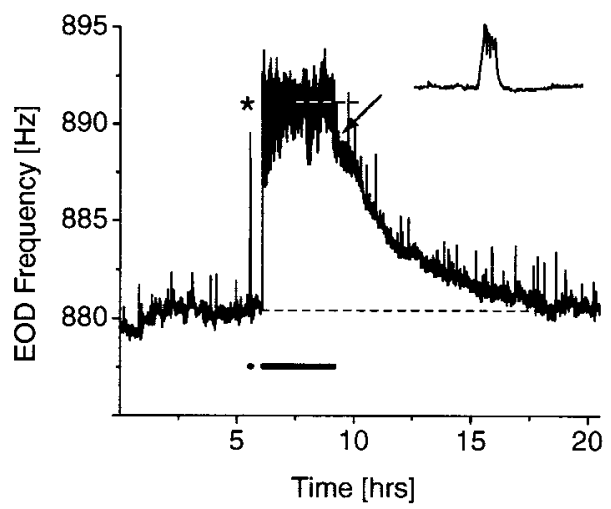

Figure 2. LTFE in the EOD frequency. $a$, Response of a female fish to a $1 \mathrm{mV} / \mathrm{cm}$ stimulus, $3 \mathrm{~Hz}$ below its EOD frequency. Initially, a 2-min-long stimulus was presented. The resulting JAR $(*)$ is enlarged in the inset. The EOD frequency relaxes back to baseline after stimulus offset. Another stimulus was presented 0.5 later for $0.5 \mathrm{hr}$. The JAR is maintained throughout the entire stimulus period and reaches a stable frequency (691.7 Hz; 5.1 $\mathrm{Hz}$ elevation; top dashed line) after 9 min. Furthermore, the EOD frequency right after stimulus offset decays rapidly to a stable but still elevated level $(2.8 \mathrm{~Hz}$ elevation; arrow), from which it gradually returns back to baseline (bottom dashed line) over $1.2 \mathrm{hr}$. The frequency and duration of the stimulus presentations are indicated by the bold line underneath the EOD frequency. $b$, Response of a male fish to a $100 \mathrm{mV} / \mathrm{cm}$ stimulus, $3 \mathrm{~Hz}$ below the EOD frequency. Again, an initial 2-min-long presentation (*) does not result in LTFE (inset), whereas a 3-hr-long stimulation (onset 0.5 hr later) results in LTFE. The initial frequency elevation is $8.5 \mathrm{~Hz}$ (arrow), and the duration until decline to baseline (bottom dashed line) is $\sim 9 \mathrm{hr}$. Note that JAR and LTFE magnitude are larger and that LTFE also lasts longer than in $a$.

throughout the in vitro recordings. The chamber consisted of an outer water bath and an inner recording well. The tissue was placed on a nylon mesh inside the recording well and perfused with continuously aerated ACSF (concentrations as described above) by means of a peristaltic pump (Dynamax RP-1; Rainin, Emeryville, CA) and vacuum-powered suction. The tissue was maintained in interface configuration and a humidified gas mixture (see above) from the outer water bath (flow rate, $81 / \mathrm{min}$ ) constantly streamed through the chamber. Gas and saline temperature were separately regulated (thermistor temperature controllers, TR-100, TCU-2; Fine Science Tools, North Vancouver, British Columbia, Canada) and kept constant at $25^{\circ} \mathrm{C}$. Recordings of the extracellular compound potential of the PMn were made with a blunt tungsten wire electrode (A \& M Systems), which was placed onto the surface of the tissue under fine control (piezoelectric inchworm drive; Burleigh, Victor, NY), and amplified over a differential AC amplifier (model 1700; A \& M Systems). The same computer system as described above was used to monitor the PMn frequency constantly. After the frequency reached sufficient stability, the tissue rostral to the PMn was stimulated through a self-made bipolar silver wire electrode. The stimulus parameters were as follows: pulse width of $400 \mu \mathrm{sec}$, three pulses spaced $25 \mathrm{msec}$ apart, train interval of $750 \mathrm{msec}$, and stimulus amplitude of $0.5 \mathrm{~mA}$ (Anapulse stimulator, model 302-T; World Precision Instruments, Sarasota, FL). The specificity of the rostral pathways in producing LTFE was tested by stimulating the tissue caudally to the PMn with the same stimulus parameters for $2 \mathrm{~min}$. This resulted in no change of the PMn frequency whatsoever (Dye, 1988) $(n=2)$. That the frequency change in the compound potential of the PMn reflected the behavior of individual cells in the syncytium was verified by recording simultaneously the compound potential extracellularly and the activity of individual cells in the PMn intracellularly with sharp electrodes $(40-60 \mathrm{M} \Omega)$. Frequencies and frequency changes were virtually the same in both recording situations $(n=$ 2 PMns).

Statistical analysis. $t$ tests were used by assuming unequal variances. All values are mean \pm SEM.

\section{RESULTS}

\section{Long-term jamming results in a prolonged JAR and poststimulus elevation of the EOD frequency}

A. leptorhynchus can only produce an upward shift in its EOD frequency during the JAR (Fig 1b) (Heiligenberg et al., 1996). In this study, individual fish were presented with sine-wave signals 3 $\mathrm{Hz}$ below their EOD frequency, which was shown previously (Bullock et al., 1972b; Dye, 1987) to elicit the highest JAR on average. First, a short 2-min-long stimulus was presented to the fish. Thirty minutes later, a longer stimulus signal of either 0.5 or $3 \mathrm{hr}$ followed. The $2 \mathrm{~min}$ stimulation served as an internal control to test the hypothesis that a longer-term stimulation is more effective in producing sensorimotor adaptation than a short stimulus presentation.

Because previous studies had only used short stimulus presentations, it was not clear whether the JAR would be maintained throughout a long-term stimulation. Our results show that the JAR is indeed continued throughout the entire stimulation (Fig. $2 a, b)$. During the long stimulus presentation, the JAR often showed an initial peak that relaxed to a steady state $(89 \%$ of the fish; $n=37$ ) (Fig. $2 a$ ). The mean frequency difference between the initial phase and the late phase of the JAR is $2.7 \pm 0.5 \mathrm{~Hz}$ $(n=37)$.

The longer stimulus was effective in eliciting a clearly visible long-term elevation in the EOD frequency (LTFE), which was apparent after the stimulus was turned off (Fig. $2 a, b$ ). Usually, the frequency gradually returned back to baseline over the time course of $0.01-8.8 \mathrm{hr}$ (see Fig. 5b), depending on the stimulus parameters. Three of 38 individuals did not show LTFE after the long stimulus presentation. In the remaining individuals, the initial 2-min-long stimulation resulted in significantly smaller LTFE than the long-term stimulation (LTFE after short stimulation was $14.2 \pm 4.7 \%$ of LTFE after long stimulation in the same individual; $n=35 ; p<0.000001$; paired $t$ test, two-tailed).

\section{Chirping does not cause behavioral LTFE}

Besides the JAR, A. leptorhynchus produces other modulations of its basic EOD frequency. Chirps are short, typically $\sim 15$-mseclong rises in EOD frequency of up to $100 \mathrm{~Hz}$ (Larimer and MacDonald, 1968; Dye, 1987; Zupanc and Maler, 1993). They are either made spontaneously or during encounters with conspecifics, in which they are thought to be aggressive or courtship signals (Hagedorn and Heiligenberg, 1985). JAR and chirps are mediated by two different prepacemaker nuclei (Heiligenberg et al., 1996). As explained in Figure 1, the JAR is controlled by the sublemniscal prepacemaker nucleus (SPPn) via NMDA receptors on relay cells, whereas chirping is produced by AMPA receptor 
Figure 3. Chirping by itself does not produce LTFE in males. $a$, Recording of a male fish's EOD frequency. An initial, 0.5-hr-long presentation of a stimulus signal $40 \mathrm{~Hz}$ above the fish's own EOD frequency outside of the jamming range elicits chirps (brief upward deflections in the frequency trace) but no upward shift in the baseline EOD frequency during or after the stimulation. However, as a control, a second stimulation, $0.5 \mathrm{hr}$ later, again for another $0.5 \mathrm{hr}$, at a frequency $3 \mathrm{~Hz}$ below the fish's EOD frequency, elicits chirps and a JAR (Fig. 1), which is followed by LTFE (arrow) after stimulus offset. Note that the temporal resolution of the signal capture was higher than in Figure 2 (20 vs $0.25 \mathrm{samples} / \mathrm{sec}$ ), therefore allowing the resolution of the brief chirps. $b$, EOD frequency change after the period of chirping alone compared with LTFE after the JAR and chirping; data are pooled from seven males, which were initially stimulated at $40 \mathrm{~Hz}$ above $(n=3)$ or $40 \mathrm{~Hz}$ below $(n=4)$ the fish's EOD frequency. $c$, Detail of the chirp at the asterisk in $a$; a chirp is characterized by a rapid EOD frequency increase, which is often accompanied by a decrease in voltage (Zupanc and Maler, 1993). The maximum frequency of the chirp is higher in $c$ than in $a$, because the frequency values in $a$ are averages over $50 \mathrm{msec}$.
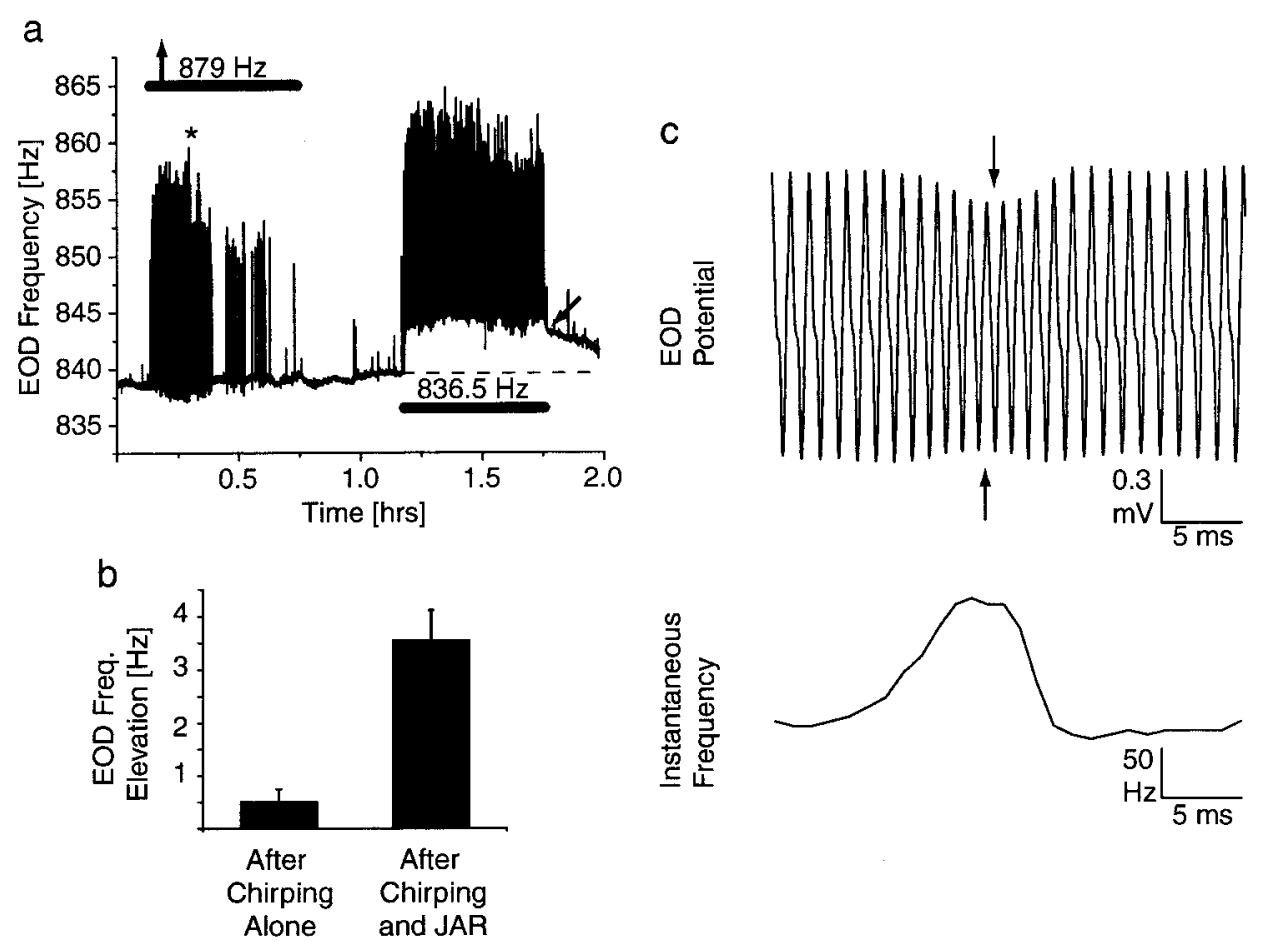

activation through input from a subportion of the thalamic prepacemaker nucleus, the PPn-C (C stands for chirping), again on relay cells. Thus, in a unique manner, because two distinct behaviors are mediated by different glutamate receptor types (Heiligenberg et al., 1996; Juranek and Metzner, 1997) (Fig. 1a-c), the involvement of a particular receptor type in eliciting LTFE can be easily studied using a simple behavioral test.

Female fish rarely chirp when tested under experimental conditions similar to ours (Dye, 1987; Zupanc and Maler, 1993; Dulka and Maler, 1994). In long-term jamming stimulations, females make at most a few dozens chirps compared with males, which often produce $>1000$ chirps throughout the stimulus presentation (J. Oestreich, unpublished observation). Both JAR and chirps are simultaneously displayed in these situations, with chirps overlaying the JAR (Fig. 1d). To test whether activation of AMPA receptors also initiates LTFE, we presented males with a stimulus frequency $40 \mathrm{~Hz}$ below $(n=4)$ or $40 \mathrm{~Hz}$ above $(n=3)$ the fish's own EOD frequency, at $1 \mathrm{mV} / \mathrm{cm}$ stimulus amplitude for $0.5 \mathrm{hr}$. In short-term presentations, this elicits only chirps (Triefenbach and Zakon, 2002) but no JAR (Fig. 1b), because it is outside of the effective jamming range (Bullock et al., 1972b; Dye, 1987). In our case, the long-term presentations also only resulted in prolonged chirping but no JAR-like elevation in base frequency during stimulation or $\operatorname{LTFE}(0.50 \pm 0.24 \mathrm{~Hz} ; n=7)$ after stimulus termination. As an internal control, $0.5 \mathrm{hr}$ later, fish were presented with a JAR signal $3 \mathrm{~Hz}$ below at the same stimulus amplitude, again for $0.5 \mathrm{hr}$, which resulted in a JAR and chirping, as well as LTFE $(3.54 \pm 0.57 \mathrm{~Hz} ; n=7)$ (Fig. 3). Thus, LTFE is not elicited by activation of AMPA receptors alone.

\section{Dependence of in vivo LTFE on stimulus duration}

Because of the decline in the initial frequency component of the JAR, we used the steady-state frequency as a measure for the JAR magnitude during the long stimulus presentation. The natural amplitude of the EOD of A. leptorhynchus varies between 0.5 and $2 \mathrm{mV} / \mathrm{cm}$ depending on the size of the fish. We chose a constant stimulus amplitude of $1 \mathrm{mV} / \mathrm{cm}$ to test the effect of stimulus duration on the JAR and LTFE magnitude. The mean magnitude of the JAR was not affected by the duration of the long stimulus presentation when compared between the $0.5(3.9 \pm 0.5$ $\mathrm{Hz} ; n=10)$ and $3(4.3 \pm 0.6 \mathrm{~Hz} ; n=9) \mathrm{hr}$ stimulated group $(p>$ 0.5 ; unpaired $t$ test, two-tailed) (Fig. 4a). Furthermore, mean LTFE in the $0.5(1.7 \pm 0.4 \mathrm{~Hz} ; n=10)$ and $3(2.3 \pm 0.5 \mathrm{~Hz} ; n=$ 9) hr stimulated groups was significantly higher than the mean LTFE in the 2-min-long stimulated group $(0.4 \pm 0.1 \mathrm{~Hz} ; n=21$; $p<0.0001$; unpaired $t$ test, two-tailed), which represented pooled data for the initial short presentations in the 0.5 and $3 \mathrm{hr}$ groups. There was no statistically significant difference between mean LTFE resulting from 0.5 - versus 3 -hr-long stimulations ( $p=$ 0.3699; unpaired $t$ test, two-tailed). These results indicate that there is a temporal threshold for activating LTFE that requires a sufficiently long enough JAR between $2 \mathrm{~min}$ to $0.5 \mathrm{hr}$ in length.

\section{Dependence of JAR and behavioral LTFE on stimulus amplitude}

Because previous work (Bullock et al., 1972b; Dye, 1987) has shown that the magnitude of the JAR is dependent on the stimulus amplitude, we asked whether the magnitude of LTFE is as well. We varied stimulus amplitude by using three groups of fish, stimulated either at $1(n=9), 15(n=9)$, or $100(n=6)$ $\mathrm{mV} / \mathrm{cm}$ and held stimulus duration constant at $3 \mathrm{hr}$ for the long-term stimulation. JAR magnitude increases with stimulus amplitude: at $1 \mathrm{mV} / \mathrm{cm}$, the mean JAR was $4.2 \pm 0.6 \mathrm{~Hz}$; at 15 $\mathrm{mV} / \mathrm{cm}$, the mean JAR was $6.4 \pm 0.6 \mathrm{~Hz}$; and, at $100 \mathrm{mV} / \mathrm{cm}$, the mean JAR was $10.5 \pm 1.2 \mathrm{~Hz}$. All differences between the means of the groups were statistically significant $(p<0.05$; unpaired $t$ test, two-tailed) (Fig. 4b).

LTFE magnitude varied with stimulus amplitude correspondingly. At $1 \mathrm{mV} / \mathrm{cm}$, the mean LTFE was $2.6 \pm 0.5 \mathrm{~Hz}$; at 15 $\mathrm{mV} / \mathrm{cm}$, mean LTFE was $4 \pm 0.8 \mathrm{~Hz}$; and, at $100 \mathrm{mV} / \mathrm{cm}$, mean 

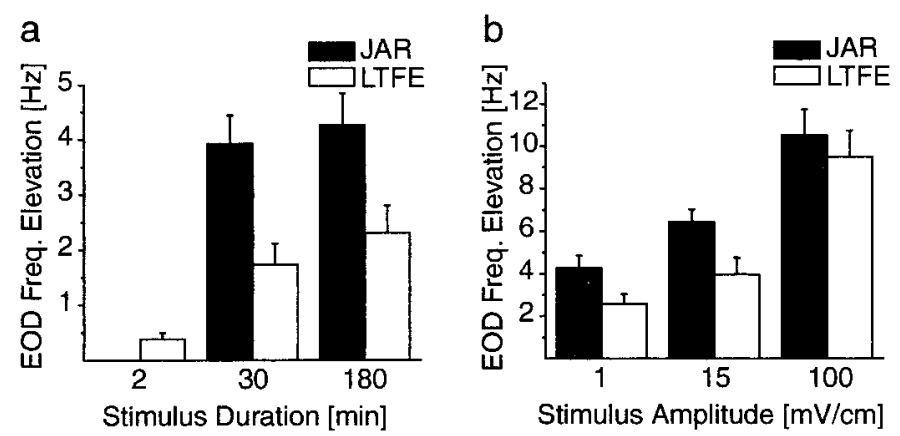

Figure 4. Dependence of JAR and LTFE magnitude on stimulus parameters. $a$, Mean changes in EOD frequency during JAR and after stimulus period (LTFE) in different groups of fish and in response to different stimulus durations at $1 \mathrm{mV} / \mathrm{cm}$ stimulus amplitude. The values were determined by measuring the elevation from baseline to stable frequency during JAR and after stimulus offset (Fig. 2). The JAR for the 2-min-long presentation was not included, because the JAR magnitude here is more similar to the initial first minutes of the JAR during the long presentations, which is usually higher than the stable frequency during these presentations and does not reflect overall magnitude of the JAR (Fig. 2b). The mean JAR for the long presentations (30 and $180 \mathrm{~min}$ ) is not affected by the stimulus duration $(30 \mathrm{~min}, n=10 ; 180 \mathrm{~min}, n=9 ; p>$ $0.5)$. Mean LTFE is significantly higher for the long presentations than for the 2-min-long presentation ( $2 \mathrm{~min}, n=21$; all comparisons, $p<0.05$ ), but the increase in mean LTFE between 30 and $180 \mathrm{~min}$ stimulated groups is not significant $(p>0.3) . b$, Mean JAR and LTFE for different stimulus amplitudes in different groups of fish at $3 \mathrm{hr}$ stimulus length for the long presentation. The JAR becomes significantly larger with increasing stimulus amplitude $(1 \mathrm{mV} / \mathrm{cm}, n=9 ; 15 \mathrm{mV} / \mathrm{cm}, n=9 ; 100 \mathrm{mV} / \mathrm{cm}$, $n=5 ; p<0.05$, all groups significantly different from each other). In correspondence, mean LTFE increases $(1 \mathrm{mV} / \mathrm{cm}, n=9 ; 15 \mathrm{mV} / \mathrm{cm}, n=$ $9 ; 100 \mathrm{mV} / \mathrm{cm}, n=6 ; p<0.05,1 \mathrm{mV} / \mathrm{cm}$ group not significantly different from $15 \mathrm{mV} / \mathrm{cm}$ group). Note that, for $100 \mathrm{mV} / \mathrm{cm}$, mean LTFE is almost as high as the JAR (Fig. 2b). $a, b$, The resting EOD frequency before onset of all stimulus experiments was fairly stable, with an average baseline frequency change of $-0.36 \pm 0.15 \mathrm{~Hz}$, measured for $3 \mathrm{hr}$ before stimulus onset $(n=31)$. The $180 \mathrm{~min}$ group in $a$ is the same than $1 \mathrm{mV} / \mathrm{cm}$ group in $b$.

LTFE was $9.5 \pm 1.3 \mathrm{~Hz}$. The difference between the means of the 1 and $100 \mathrm{mV} / \mathrm{cm}$ groups was significant, as were the 15 and 100 $\mathrm{mV} / \mathrm{cm}$ groups ( $p<0.05$; unpaired $t$ test, two-tailed). The 1 and $15 \mathrm{mV} / \mathrm{cm}$ groups were not significantly different $(p>0.05)$. In contrast to the larger difference between JAR and LTFE magnitude in the other groups, at $100 \mathrm{mV} / \mathrm{cm}$, the mean LTFE magnitude almost becomes as large as the mean JAR magnitude. If the JAR magnitude is a measure of the amount of afferent drive to the PMn and LTFE is a result of it, then the diminished difference possibly indicates a saturation of the cellular mechanisms, which underlie LTFE.

\section{Quantitative features of JAR and LTFE}

Because there was no significant difference between the 0.5 and 3 hr stimulated groups in terms of JAR and LTFE magnitude and because JAR magnitude and LTFE magnitude both varied with a given stimulus amplitude, we plotted LTFE as a function of JAR magnitude for all long-term stimulated groups of all stimulus amplitudes (Fig. 5a). Regression analysis showed a significant correlation between these parameters $(r=0.95 ; p<0.0001 ; n=$ $40 ; y=0.94 x-1.77)$. The intercept of the regression line at 1.9 $\mathrm{Hz}$ for the JAR indicated that, in addition to the temporal threshold, there is a further threshold for attaining LTFE; a minimum JAR magnitude is required to activate the cellular mechanisms for LTFE.

To test whether LTFE magnitude predicted LTFE duration, we performed a regression analysis on fish with a regular EOD frequency after stimulus termination $(n=18)$ (Fig. $5 b)$. A significant number of fish $(n=23)$ showed a more irregular EOD frequency sometime after the stimulus signal was turned off, which stemmed from another spontaneously displayed EOD modulation of unknown function, so-called yodels. Yodels can also contribute to a long-term resetting of the EOD frequency, overlaying the LTFE caused by JAR (Oestreich et al., 1999). Those fish were excluded from this analysis. In the remaining fish, LTFE could be clearly followed for $0.01-8.8 \mathrm{hr}$. The higher the initial LTFE magnitude, the longer the EOD frequency stayed elevated above the prestimulus baseline frequency $(r=0.84 ; p<$ $0.0001 ; n=18 ; y=0.6 x+0.19)$.

There was no obvious difference between females and males in JAR magnitude or LTFE for both relationships (Fig. $5 a, b$ ). The observation that males chirp during jamming and females do not suggests that activation of AMPA receptors in conjunction with NMDA receptors has no effect on LTFE and agrees with our previous demonstration that, in males, chirping alone does not initiate LTFE (Fig. 3). Therefore, only NMDA receptor activation seems to contribute to LTFE initiation.

\section{LTFE in the in vitro preparation of the PMn}

The PMn forms an ovoid protrusion from the ventral surface of the brainstem and can be easily excised. It remains viable for several hours in a tissue slice chamber and continues to discharge at a rate correlated to the fish's original EOD frequency (Meyer, 1984; Schaefer and Zakon, 1996; Smith and Zakon, 2000) (Oestreich, unpublished observation).

In previous work (Dye, 1988; Dye et al., 1989), LTFE was induced in the slice preparation of the isolated PMn with only the terminal portion of the afferent fibers attached. A brief, tetanic stimulation of the fiber pathways resulted in temporary activation of the afferent input and was followed by a step-up of the PMn frequency. However, only a few seconds of recordings were shown, and the statement was made the effect only lasted for seconds. Furthermore, these studies were not related to possible long-term effects of sustained activation of afferent input to the PMn on the EOD frequency in vivo. Additionally, a brief, intense tetanic stimulation does not mimic the prolonged, low-frequency activity of the afferent input expected in presence of a long-term jamming situation. To test whether the PMn indeed could be the locus of change in the electromotor circuit, we also turned to the slice preparation. Unfortunately, nothing is known about the natural firing rates of the prepacemaker nuclei. However, we developed a stimulation paradigm that mimicked an afferent firing pattern that caused a JAR-like PMn frequency elevation.

Before any stimulation, excised PMns were allowed to recover in a tissue chamber while we constantly monitored the extracellular compound potential and frequency of the PMn. The PMns were often quiescent for up to $30 \mathrm{~min}$, until neural activity set in relatively suddenly within a few seconds. The PMn frequency usually ramped up for another hour to $2 \mathrm{hr}$ until it reached stability within a few Hertz (Oestreich, unpublished observation). Thirty minutes of relative stability were sought before stimulation was attempted. A 20-min-long stimulation at $1.5 \mathrm{~Hz}$ resulted in a progressive increase of the PMn frequency during the stimulation, which, in some cases, was followed by a slight decrease until a stable frequency was reached (Fig. 6). After stimulus termination, the PMn frequency rapidly declined to a lower level, which, however, was still elevated above the original baseline frequency. 
Figure 5. The expression of LTFE is positively correlated with the JAR magnitude, and LTFE duration in turn is dependent on the LTFE magnitude. $a$, Linear fit of the relationship between JAR and LTFE magnitude for male $(n=17)$ and female $(n=23)$ fish (total, $n=40 ; r=0.95 ; p<0.0001)$. $b$, Linear fit for LTFE magnitude versus LTFE duration. Only fish with regular EOD frequency over a longterm period after stimulus offset were included in this analysis (males, $n=8$; females, $n=10$; total, $n=18 ; r=0.84 ; p<0.0001)$. In $a$ and $b$, no obvious difference between males and females with respect to JAR and LTFE is noticeable, despite the fact that males are also chirping during the JAR. Both data sets were generated from the responses of all long-term stimulations regardless of stimulus amplitude.

a

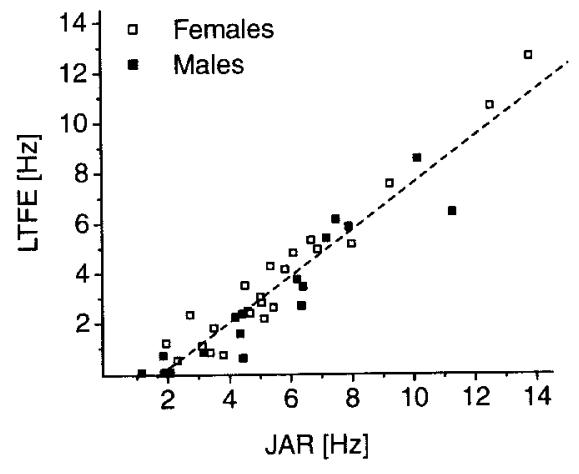

b

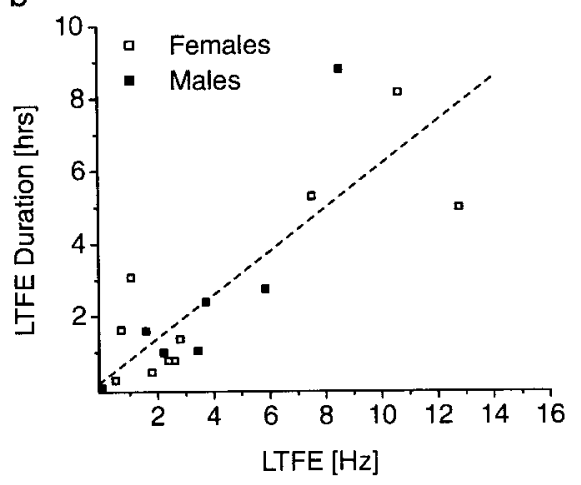

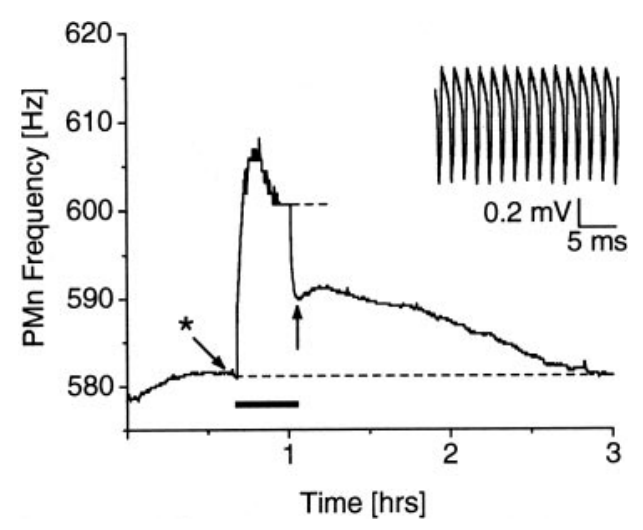

Figure 6. LTFE in a preparation of the isolated PMn. After recording a stable baseline frequency for $\sim 0.5 \mathrm{hr}$, low-frequency stimulation (duration indicated by bold line) of the afferent fibers for 20 min resulted in a progressive increase in PMn frequency until a stable frequency (top dashed line) was reached. Stimulus offset led to an initial rapid decline in frequency to a point (arrow), which was still elevated above baseline (bottom dashed line). LTFE lasted for $1.9 \mathrm{hr}$. The inset shows a trace of the extracellularly recorded compound potential of the PMn at the asterisk.

The frequency then only gradually returned back to baseline over a time course of up to $2.8 \mathrm{hr}$ (Fig. $7 c$ ).

In conclusion, the behavior of the PMn frequency in vitro shows a striking resemblance to the development of the EOD frequency during and after a prolonged stimulus presentation, suggesting that a resetting of the PMn indeed underlies the EOD frequency change.

\section{Dependence of in vitro LTFE on stimulus duration}

To further test this notion, we investigated whether the expression of LTFE in the slice is also affected by the stimulus duration as it is in vivo (Fig. 4a). We used low-frequency stimulation at 1.5 Hz over $2(n=6)$ and $20(n=7)$ min in groups of different PMns. We chose a $20 \mathrm{~min}$ stimulation instead of $30 \mathrm{~min}$ as in one of the in vivo groups, because of an increased probability of run-down effects with time.

Mean LTFE in the 2 min group was $4.8 \pm 0.8 \mathrm{~Hz}$, whereas stimulation over $20 \mathrm{~min}$ resulted on average in $12.5 \pm 3.5 \mathrm{~Hz}$ LTFE. These values were significantly different ( $p<0.05$; unpaired $t$ test, one-tailed) (Fig. 7a). LTFE in both groups however was higher than in in vivo, suggesting that the afferent firing rate and/or number of recruited fibers in vitro was different from the situation in the intact animal. In summary, these data together with the findings in vivo (Fig. 4a) imply a temporal threshold for activation of the LTFE mechanism.

\section{Quantitative features of PMn frequency during stimulation and LTFE in vitro}

The elevation in PMn frequency during stimulation should reflect the strength of the afferent drive of the prepacemaker fiber pathways to the PMn and represents the equivalent of a JAR in vitro. Afferent activity should only last as long as the stimulation is ongoing, because the somata of the prepacemaker neurons are not included in the slice preparation. Therefore, any frequency elevation after stimulus termination should be the result of changes at the postsynaptic site, the PMn. Furthermore, the magnitude of the postsynaptic change, as it is reflected by LTFE, should be correlated with the strength of the synaptic input, which, as just pointed out, is represented in the PMn frequency elevation during stimulation. To test whether the relationship between JAR as a measure of presynaptic activity and LTFE as a postsynaptic result in vivo (Fig. $5 a$ ) is also reflected in the isolated PMn, we used data from two different stimulus groups for a regression analysis, in which LTFE is plotted against the PMn frequency during stimulation $(r=0.88 ; p<0.0001 ; n=14 ; y=$ $0.48 x-3.18$ ) (Fig. 7b). A low-frequency stimulation at $1.5 \mathrm{~Hz}$ frequency using a triple-pulse bout (individual pulses spaced 25 msec apart) produces a relatively large frequency increase during stimulation and also results in high LTFE $(n=9)$. Although there is considerable scatter in the data using the same stimulation paradigm (triple pulse), we did not obtain weaker responses to explore possible threshold effects seen in vivo (Fig. 5a), in which pooling of groups stimulated with several different parameter combinations generated enough variability. Therefore, we included data from a second group of PMns, which were stimulated at the same frequency, but only with a single pulse. This resulted in a lower PMn frequency elevation during stimulation, which almost produces no LTFE $(n=5)$. These data further support the idea that the afferent input to the PMn has to be of sufficient magnitude to activate the LTFE signaling pathway. The threshold is $6.6 \mathrm{~Hz}$, which is higher than the threshold in vivo (1.9 $\mathrm{Hz}$ ). Again, there are no differences between male and female PMns. An analysis of in vitro LTFE duration versus LTFE magnitude (in vivo; Fig. $5 b$ ) also resulted in a strong correlation $(r=0.9 ; p<0.001 ; n=11 ; y=6.3 x+17.5)$ (Fig. $7 c)$, further strengthening the hypothesis that LTFE in the PMn underlies behavioral LTFE. 
a

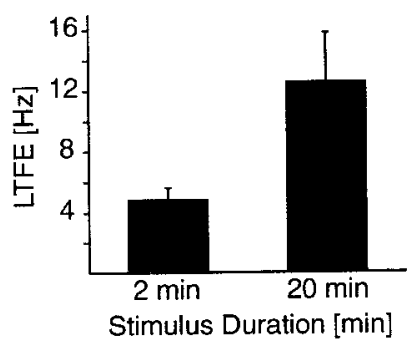

b

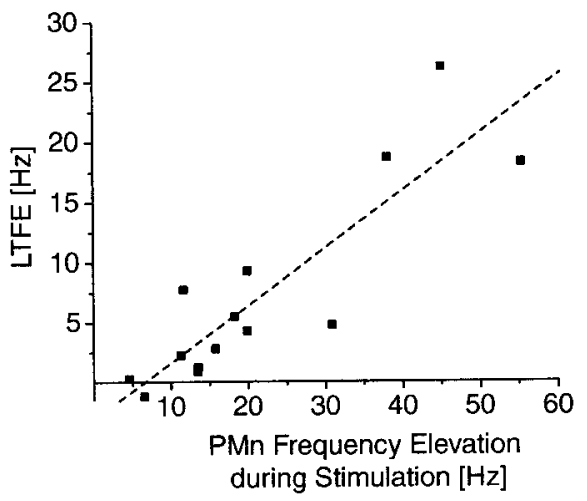

$c$

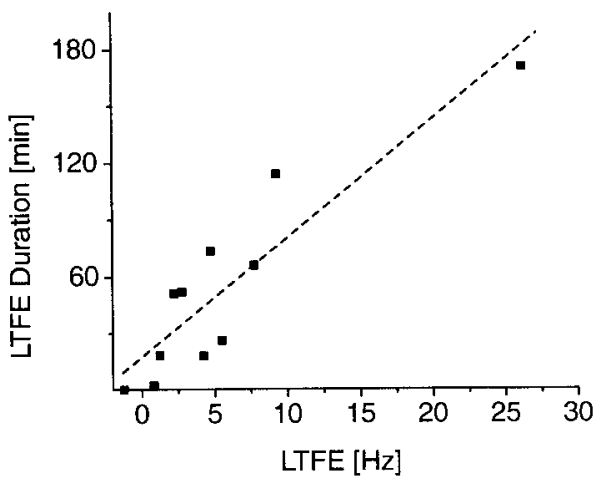

Figure 7. Quantitative features of LTFE in vitro. a, A low-frequency stimulation of the afferent fiber at $1.5 \mathrm{~Hz}$ using triple pulses spaced $25 \mathrm{msec}$ apart results in lower LTFE for 2-min-long stimulations $(n=6)$ than for 20-min-long presentations $(n=7 ; p<0.05)$. Note that LTFE in the isolated PMn preparation in both in vitro groups is higher than in vivo. $b$, Correlation between LTFE and PMn frequency elevation during stimulation for pooled data of the 20 min group in $a$ and a group of PMns $(n=5)$ that were stimulated with a single pulse instead of triple pulses (total, $n=14 ; r=0.88 ; p<0.0001)$. $c$, LTFE duration as a function of LTFE magnitude $(n=11 ; r=0.9 ; p<0.001)$. a-c, Note that all in vitro relationships resemble their in vivo counterparts.

\section{DISCUSSION}

Our findings demonstrate that long-lasting electrosensory input leads to sensorimotor adaptation in A. leptorhynchus. The adaptation is a stable change in the frequency of the electromotor output (the EOD), which becomes noticeable after the stimulus is switched off. It is likely that the basis for this adaptation is a long-term NMDA receptor-dependent resetting of the spike frequency in the electromotor command nucleus (the PMn). The magnitude of the adaptive change is correlated with the amount of synaptic input received by the postsynaptic neurons.

\section{Sensorimotor adaptation is produced by a synaptically induced increase in postsynaptic firing rate}

If no jamming signal is present, the PMn will discharge at its basic rate and the EOD frequency is stable. In the presence of a jamming signal, the synaptic input to the SPPn from higher electrosensory regions drives the SPPn to fire faster, which in turn increases the firing frequency of the PMn and, with that, the EOD frequency (Heiligenberg et al., 1996). This increase in EOD frequency is the JAR (Fig. 1a,b). Initially, the responsibility of maintaining the JAR is with the presynaptic nucleus, the SPPn. The behavioral output of the electromotor system now becomes the crucial link, which closes the feedback loop. By raising its own EOD frequency away from the jamming frequency, the fish will decrease interference, subsequently reducing the sensory input feeding into the SPPn. However, with long-lasting synaptic input, a cellular mechanism is induced in the PMn, which leads to a persistent increase in the activity of the PMn. This situation then allows for a decrease in the synaptic activity, because the responsibility of maintaining the increased EOD frequency during the JAR is now with the postsynaptic site, the PMn (Fig. 8a,b).

What is the need for adaptation in the frequency of the electromotor output? The alternative is for continued synaptic input and, possibly, a prolonged activation of NMDA receptors during long-term sensory stimulation. NMDA receptors conduct $\mathrm{Ca}^{2+}$ (Gasic and Hollmann, 1992), and one possible negative consequence of an extensive and long-term $\mathrm{Ca}^{2+}$ influx is excitotoxicity (Choi, 1988; Rothman and Olney, 1995; DeLorenzo et al., 1998). This possibility is supported by the fact that, in a few preparations, the stimulation irreversibly resulted in a progres- sive, apparent uncoupling of PMn neurons and a reduction of the PMn potential (Oestreich, unpublished observations), consistent with the effect of aspartate bath application or treatment with a $\mathrm{Ca}^{2+}$ ionophore (Dye, 1991).

\section{Positive feedback at the cellular level allows for negative feedback on the systems level}

An emerging theme in cellular neuroscience is that neurons adapt to long-lasting synaptic stimulation by downregulating their intrinsic excitability, for example, to adapt to a sustained stimulus for enhancement of novelties in the sensory stream (Laughlin, 1989; Sanchez-Vives et al., 2000a,b; McCormick, 2001), to avoid overstimulation, or the saturation of firing rates. The last situation is termed homeostatic synaptic plasticity (Turrigiano and Nelson, 2000). Here, global synaptic strength and/or membrane excitability are turned down in response to continuing excitatory input to prevent saturation of the firing rate by ever increasing correlation between presynaptic and postsynaptic firing and hence runaway in increasing synaptic strength. A few studies have shown longlasting increases in postsynaptic activity after relatively short, but high-frequency synaptic stimulation (Alkon, 1984; Kaczmarek et al., 1986; de Jonge et al., 1990; Aizenman and Linden, 2000) or bath application of neurotransmitter receptor agonists (Sivilotti and Nistri, 1992; Chu and Hablitz, 2000) in vitro. Our results add that, even with long, low-frequency electrical stimulations over 20 min, neurons can respond with a long-lasting net enhancement in their activity. This positive coupling between synaptic inputs and postsynaptic excitability is non-homeostatic control of neuronal excitability, because the set point of neuronal activity is changed in a stable manner. Homeostatic control of neuronal excitability by definition keeps the postsynaptic firing rate at a specific set point, which is under internal cellular control. We propose that at least some neurons (i. e. the neurons in the PMn) can be set to fire at various firing rates in a certain "healthy" (i.e., non-excitotoxic or nonsaturated) range of possible firing rates. These neurons adjust their firing rate in this range depending on the requirements of the circuit in which they are embedded. Therefore, the firing rate of the neuron in this range is set externally by the circuit and not internally. A detailed understanding of the exact role of these neurons in their circuits or the relationship of these 
a

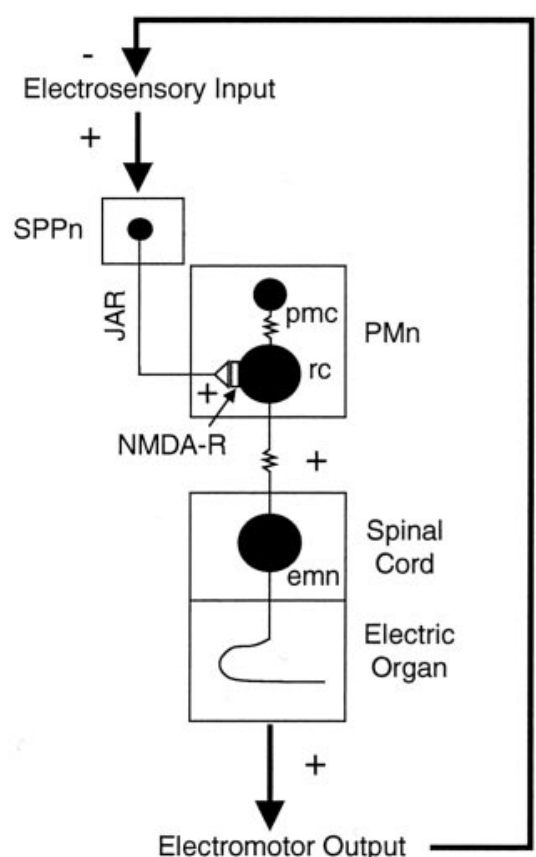

b

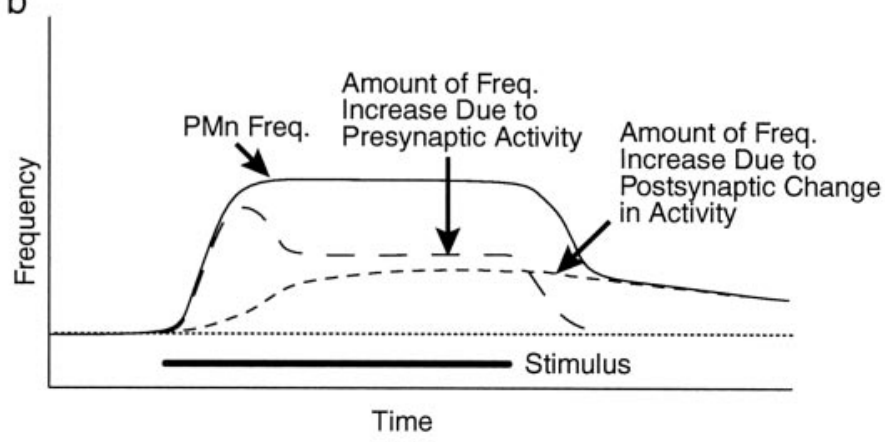

C

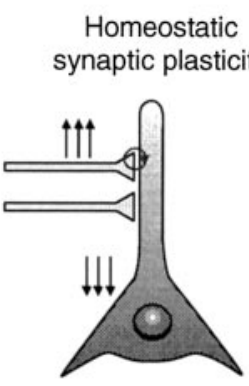

Situation in the PMn

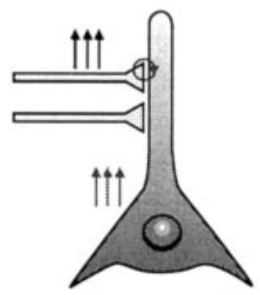

Figure 8. Positive feedback at the cellular level results in negative feedback on the circuit level. $a$, Electromotor circuitry with sensory input and motor output. An increase in the EOD frequency during the JAR effectively reduces jamming of the electrosensory system and therefore reduces the sensory input feeding back into the electromotor system. $b$, Model of presynaptic and postsynaptic influence on the PMn frequency. In the initial phase of the JAR, the frequency increase is attributable to presynaptic drive by the SPPn. Continuing activation of NMDA receptors results in the induction of a postsynaptic signaling mechanism, which leads to an increase in the PMn firing rate. Synaptic input ceases after stimulus termination, and the resetting of the PMn becomes visible as LTFE. $c$, In homeostatic synaptic plasticity, neurons adapt to sustained synaptic activation (upward arrows) by turning down their excitability (downward arrows) or synaptic strength globally to avoid a saturation of their firing rate by increasing correlation between presynaptic and postsynaptic firing and hence runaway in increasing synaptic strength (Turrigiano and Nelson, 2000) (circle). The situation in the PMn is different: a sustained increase in synaptic input results in a persistent increase in postsynaptic firing rate. However, this can be understood if the entire circuit is considered $(a)$.

circuits to behavior is helpful in interpreting in vitro observations of stable changes in neuronal firing rate.

In this paper, we present a novel example of a synaptically induced long-lasting increase in postsynaptic excitability, an example of positive coupling between synaptic stimulation and postsynaptic excitability. However, when considered in the context of the complete functioning circuit and including the behavioral link in the feedback loop, it is clear that positive feedback at the cellular level results in negative feedback on the circuit and organismic level, thus actually contributing to, rather than conflicting with, homeostatic adjustment of the circuit.

\section{Relationships between LTFE and stimulus parameters in vivo and in vitro}

A key problem in sensorimotor adaptation is identifying the locus of the change. Our experiments suggest that sensorimotor adaptation in the electromotor system can be localized to the neurons of the pacemaker nucleus, because the characteristics of the changes that we observe in the slice closely approximate those observed in the behaving animal. Mainly, the magnitude and duration of the JAR predicts the magnitude and duration of the behavioral LTFE similarly to the way that the magnitude and duration of the fictive JAR predicts the magnitude and duration of LTFE in vitro. Furthermore, our results show that both behavioral LTFE and LTFE in vitro have magnitude and temporal thresholds: they do not occur unless the JAR is of sufficient magnitude $(\sim 2 \mathrm{~Hz}$ in vivo $)$ and duration $(>2 \mathrm{~min})$.
LTFE can be considered a form of neuronal memory. The strength and duration of that memory, as measured by the magnitude and duration of the elevation of EOD frequency, is correlated with the duration and the amplitude of the sensory stimulus. This relationship predicts that LTFE occurs in a graded manner and suggests that the underlying cellular mechanisms are working in a linear range until the PMn frequency is appropriately adjusted. This mechanism must have a temporal and intensity threshold and be capable of a time-intensity tradeoff such that total stimulus strength (stimulus duration $\times$ magnitude) is integrated.

\section{Receptor pharmacology}

Previous work has demonstrated (Dye et al., 1989; Heiligenberg et al., 1996) that a short JAR is mediated by NMDA receptors as the NMDA receptor antagonist D-APV abolishes the JAR completely. Additionally, one of the previous studies (Dye et al., 1989) shows that in vitro LTFE induced by short, tetanic stimulation of the afferent fibers is also blocked by D-APV. The possibility exists that LTFE as the result of long-term low-frequency stimulation could be at least partially attributable to the corelease of another amino acid, peptidergic neurotransmitter, or activation of metabotropic glutamate receptors. However, preliminary experiments indicate that fictive JAR and LTFE in vitro during a long-term, low-frequency stimulation are also effectively blocked by the NMDA receptor antagonists (+)-MK-801 [(+)-5-methyl-10,11dihydro-5H-dibenzo [a,d] cyclohepten-5,10-imine hydrogen mal- 


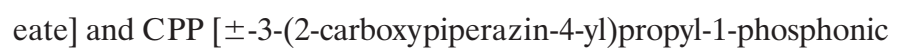
acid] (Oestreich, unpublished observation). Together, these pharmacological data suggest that even a long-term JAR is mediated by NMDA receptors and that the JAR itself is the prerequisite for LTFE.

The behavioral data presented here show that chirping in males did not produce LTFE (Fig. 3). Furthermore, LTFE did not vary between males and females, despite the fact that males chirp vigorously while performing a JAR, whereas females do not (Fig. 5). These results therefore indicate that AMPA receptors play no role in LTFE either when activated alone or coactivated with NMDA receptors.

\section{Cellular mechanisms for LTFE}

The quantitative correlation between synaptic strength and LTFE magnitude could be based on the amount of $\mathrm{Ca}^{2+}$ influx through NMDA receptors into the cell. By using the intracellular $\mathrm{Ca}^{2+}$ concentration, the PMn neurons would measure the duration and strength of exposure to a signal intruding on the sensory space and adjust their frequency shift appropriately to avoid jamming. What are some of the possible, $\mathrm{Ca}^{2+}$-activated mechanisms for LTFE?

So far, most work on molecular mechanisms for the engram of memory has focused on hebbian plasticity, in which the memoryinducing, chemical synapse itself becomes the substrate for change (Bliss and Collingridge, 1993; Marder et al., 1996; Spitzer, 1999). However, more and more evidence is supporting the involvement of changes at other cellular sites in memory, particularly changes in network connectivity, either at synapses different from the inducing chemical synapse and/or changes at gap junctions. A postsynaptic network can be synaptically induced to maintain an image of that input by a persistent change in activity, for example by recurrent excitation (Aksay et al., 2001), or by changing levels of tonic inhibition.

In support of a possible role of gap junctions in LTFE, evidence at the mixed synapse of the auditory nerve with the Mauthner cell in goldfish has shown that $\mathrm{Ca}^{2+}$ influx via NMDA receptors can modulate the conductivity of gap junctions in a long-term manner (Pereda and Faber, 1996; Pereda et al., 1998), and network models (Kepler et al., 1990) have indicated that changes in the number of open gap junctions could also increase the frequency in a network of electrotonically coupled neurons. The neurons in the PMn are highly coupled (Bennett et al., 1967; Elekes and Szabo, 1985; Moortgat et al., 2000), and, therefore, the possibility exists that a change in electrotonic connectivity underlies LTFE.

Alternatively, the intrinsic excitability of the individual neurons could be affected in a long-term manner by synaptic input (Alkon, 1984; de Jonge et al., 1990; Marder et al., 1996; Desai et al., 1999; Spitzer, 1999; Aizenman and Linden, 2000). $\mathrm{Ca}^{2+}$ influx through activated NMDA receptors has been linked to changes in membrane currents (Puro et al., 1996; DeLorenzo et al., 1998; Aizenman and Linden, 2000), and a possible candidate target in the PMn is a potassium current. Previous work (Dye, 1991; Smith and Zakon, 2000) has shown that the potassium channel blocker 4-AP has a profound influence on the activity of the PMn by raising the firing frequency. Among others, these findings would be in correspondence with Kv3.3, which is present in the PMn (R. Turner, personal communication). Kv3.3 is an A-type current known to influence the interspike interval and, therefore, setting the spike rate in other systems (Smith, 1999). That this situation could be a universal mechanism is supported by data (Wu and Barish, 2000) in the hippocampus, which show that an increase in intracellular $\mathrm{Ca}^{2+}$ concentration reduces a 4-AP-sensitive potassium current and leads to increased excitability.

The question of which cellular mechanism is involved in producing LTFE will be addressed in future studies.

\section{REFERENCES}

Aizenman CD, Linden DJ (2000) Rapid, synaptically driven increases in the intrinsic excitability of cerebellar deep nuclear neurons. Nat Neurosci 3:109-111.

Aksay E, Gamkrelidze G, Seung HS, Baker R, Tank DW (2001) In vivo intracellular recording and perturbation of persistent activity in a neural integrator. Nat Neurosci 4:184-193.

Alkon DL (1984) Changes of membrane currents during learning. J Exp Biol 112:95-112.

Bennett MVL, Pappas GD, Gimenez M, Nakajima Y (1967) Physiology and ultrastructure of electrotonic junctions. IV. Medullary electromotor nuclei in gymnotid fish. J Neurophysiol 30:236-300.

Bliss TVP, Collingridge GL (1993) A synaptic model of memory: longterm potentiation in the hippocampus. Nature 361:31-39.

Bullock TH, Hamstra RH, Scheich H (1972a) The jamming avoidance response of high-frequency electric fish. I. General features. J Comp Physiol 77:1-22.

Bullock TH, Hamstra RH, Scheich H (1972b) The jamming avoidance response of high frequency electric fish. II. Quantitative aspects. J Comp Physiol 77:23-48.

Choi DW (1988) Glutamate neurotoxicity and diseases of the nervous system. Neuron 1:623-634.

Chu Z, Hablitz JJ (2000) Quisqualate induces an inward current via mGluR activation in neocortical pyramidal neurons. Brain Res 879:88-92.

de Jonge MC, Black J, Deyo RA, Disterhoft JF (1990) Learninginduced afterhyperpolarization reductions in hippocampus are specific for cell type and potassium conductance. Exp Brain Res 80:456-462.

DeLorenzo RJ, Pal S, Sombati S (1998) Prolonged activation of the $N$-methyl-D-aspartate receptor-Ca ${ }^{2+}$ transduction pathway causes spontaneous recurrent epileptiform discharges in hippocampal neurons in culture. Proc Natl Acad Sci USA 95:14482-14487.

Desai NS, Rutherford LC, Turrigiano GG (1999) Plasticity in the intrinsic excitability of cortical pyramidal neurons. Nat Neurosci 2:515-520.

Dulka JG, Maler L (1994) Testosterone modulates female chirping behavior in the weakly electric fish, Apteronotus leptorhynchus. J Comp Physiol [A] 174:331-343.

Dunlap KD, Smith GT, Yekta A (2000) Temperature dependence of electrocommunication signals and their underlying neural rhythms in the weakly electric fish, Apteronotus leptorhynchus. Brain Behav Evol 55:152-162.

Dye J (1987) Dynamics and stimulus-dependence of pacemaker control during behavioral modulations in the weakly electric fish, Apteronotus. J Comp Physiol [A] 161:175-185.

Dye J (1988) An in vitro physiological preparation of a vertebrate communicatory behavior: chirping in the weakly electric fish, Apteronotus. J Comp Physiol [A] 163:445-458.

Dye J (1991) Ionic and synaptic mechanisms underlying a brainstem oscillator: an in vitro study of the pacemaker nucleus of Apteronotus. J Comp Physiol [A] 168:521-532.

Dye J, Heiligenberg W, Keller CH, Kawasaki M (1989) Different classes of glutamate receptors mediate distinct behaviors in a single brainstem nucleus. Proc Natl Acad Sci USA 86:8993-8997.

Elekes K, Szabo T (1985) Synaptology of the medullary command (pacemaker) nucleus of the weakly electric fish (Apteronotus leptorhynchus) with particular reference to comparative aspects. Exp Brain Res 60:509-520.

Gasic GP, Hollmann M (1992) Molecular neurobiology of glutamate receptors. Annu Rev Physiol 54:507-536.

Hagedorn M, Heiligenberg W (1985) Court and spark: electric signals in the courtship and mating of gymnotid fish. Anim Behav 33:254-265.

Heiligenberg W (1986) Jamming avoidance responses. In Electroreception (Bullock TH, Heiligenberg W, eds), pp 613-649. New York: Wiley

Heiligenberg W, Metzner W, Wong CJ, Keller CH (1996) Motor control of the jamming avoidance response of Apteronotus leptorhynchus: evolutionary changes of a behavior and its neuronal substrates. J Comp Physiol [A] 179:653-674.

Held R, Freedman SJ (1963) Plasticity in human sensorimotor control. Science 142:455-462.

Juranek J, Metzner W (1997) Cellular characterization of synaptic modulations of a neuronal oscillator in electric fish. J Comp Physiol [A] 181:393-414.

Kaczmarek LK, Strong JA, Kauer JA (1986) The role of protein kinases in the control of prolonged changes in neuronal excitability. Prog Brain Res 69:77-90. 
Kepler TB, Marder E, Abbott LF (1990) The effect of electrical coupling on the frequency of model neuronal oscillators. Science 248:83-85.

Larimer JL, MacDonald JA (1968) Sensory feedback from electroreceptors to electromotor pacemaker centers in gymnotids. Am J Physiol 214:1253-1261.

Laughlin SB (1989) The role of sensory adaptation in the retina. J Exp Biol 146:39-62.

Marder E, Abbott LF, Turrigiano GG, Liu Z, Golowasch J (1996) Memory from the dynamics of intrinsic membrane currents. Proc Natl Acad Sci USA 93:13481-13486.

McCormick DA (2001) Brain calculus: neural integration and persistent activity. Nat Neurosci 4:113-114.

Meyer H (1984) Steroid influences upon discharge frequencies of intact and isolated pacemakers of weakly electric fish. J Comp Physiol [A] 154:659-668.

Moortgat KT, Bullock TH, Sejnowski TJ (2000) Precision of the pacemaker nucleus in a weakly electric fish: network versus cellular influences. J Neurophysiol 83:971-983.

Oestreich J, Zakon HH, Larimer JL (1999) Behavioral correlates for synaptically induced changes in the pacemaker nucleus frequency of a weakly electric fish. Soc Neurosci Abstr 25:1362.

Pereda AE, Faber DS (1996) Activity-dependent short-term enhancement of intercellular coupling. J Neurosci 16:983-992.

Pereda AE, Bell TD, Chang BH, Czernik AJ, Nairn AC, Soderling TR, Faber DS (1998) $\mathrm{Ca}^{2+} /$ calmodulin-dependent kinase II mediates simultaneous enhancement of gap-junctional conductance and glutamatergic transmission. Proc Natl Acad Sci USA 95:13272-13277.

Puro DG, Yuan JP, Sucher NJ (1996) Activation of NMDA receptorchannels in human retinal Muller glial cells inhibits inward-rectifying potassium currents. Vis Neurosci 13:319-326.

Rothman SM, Olney JW (1995) Excitotoxicity and the NMDA receptor-still lethal after eight years. Trends Neurosci 18:57-58.

Sanchez-Vives MV, Nowak LG, McCormick DA (2000a) Membrane mechanisms underlying contrast adaptation in cat area 17 in vivo. J Neurosci 20:4267-4285.

Sanchez-Vives MV, Nowak LG, McCormick DA (2000b) Cellular mechanisms of long-lasting adaptation in visual cortical neurons in vitro. J Neurosci 20:4288-4299.
Schaefer JE, Zakon HH (1996) Opposing actions of androgen and estrogen on in vitro firing frequency of neuronal oscillators in the electromotor system. J Neurosci 16:2860-2868.

Sivilotti L, Nistri A (1992) An intracellular study of the effects of GABA on frog tectal neurones in vitro. Neurosci Lett 145:28-32.

Smith GT (1999) Ionic currents that contribute to a sexually dimorphic communication signal in weakly electric fish. J Comp Physiol [A] 185:379-387.

Smith GT, Zakon HH (2000) Pharmacological characterization of ionic currents that regulate the pacemaker rhythm in a weakly electric fish. J Neurobiol 42:270-286.

Smith GT, Lu Y, Zakon HH (2000) Parvocells: a novel interneuron type in the pacemaker nucleus of a weakly electric fish. J Comp Neurol 423:427-439.

Spitzer NC (1999) New dimensions of neuronal plasticity. Nat Neurosci 2:489-491.

Takizawa Y, Rose GJ, Kawasaki M (1999) Resolving competing theories for control of the jamming avoidance response: the role of amplitude modulations in electric organ discharge decelerations. J Exp Biol 202:1377-1386.

Triefenbach FA, Zakon HH (2002) Effects of sex sensitivity and status on cue recognition in the weakly electric fish, Apteronotus leptorhynchus. Anim Behav, in press.

Turrigiano GG, Nelson SB (2000) Hebb and homeostasis in neuronal plasticity. Curr Opin Neurobiol 10:358-364.

Watanabe A, Takeda K (1963) The change of discharge frequency by AC stimulus in a weakly electric fish. J Exp Biol 40:57-66.

Wu R-L, Barish ME (2000) Calcium regulation of the slowlyinactivating voltage-gated potassium current $\left(I_{\mathrm{d}}\right)$ of hippocampal pyramidal neurons. Soc Neurosci Abstr 26:337.2.

Zupanc GKH, Maler L (1993) Evoked chirping in the weakly electric fish Apteronotus leptorhynchus: a quantitative biophysical analysis. Can J Zool 71:2301-2310.

Zupanc GKH, Maler L (1997) Neuronal control of behavioral plasticity: the prepacemaker nucleus of weakly electric gymnotiform fish. J Comp Physiol [A] 180:99-111. 